Acta Crystallographica Section E

Structure Reports

Online

ISSN 1600-5368

Peter Kirsop, John M. D. Storey and William T. A. Harrison*

Department of Chemistry, University of Aberdeen, Meston Walk, Aberdeen AB24 3UE, Scotland

Correspondence e-mail:

w.harrison@abdn.ac.uk

\section{Key indicators}

Single-crystal X-ray study

$T=120 \mathrm{~K}$

Mean $\sigma(\mathrm{C}-\mathrm{C})=0.006 \AA$

$R$ factor $=0.052$

$w R$ factor $=0.084$

Data-to-parameter ratio $=19.1$

For details of how these key indicators were automatically derived from the article, see http://journals.iucr.org/e.

\title{
Ethyl 3,5-bis(allyloxy)-4-bromobenzoate
}

The asymmetric molecular conformation of the title compound, $\mathrm{C}_{15} \mathrm{H}_{17} \mathrm{BrO}_{4}$, may be be influenced by an intramolecular $\mathrm{C}-\mathrm{H} \cdots \mathrm{O}$ interaction. The molecules form $\pi-\pi$ stacks in the crystal structure.

\section{Comment}

The title compound, (I) (Fig. 1), was prepared as part of our studies to determine the philicity of aryl radicals by competitive cyclization reactions (Kirsop et al., 2004).<smiles>C=CCOc1cc(C(=O)OCC)cc(OCC=C)c1Br</smiles>

(I)

Compound (I) possesses normal geometrical parameters. The dihedral angle between the mean plane of the C1-C6 benzene ring and the plane of the $\mathrm{C} 7 / \mathrm{O} 1 / \mathrm{O} 2$ group is $6.0(5)^{\circ}$. The two $-\mathrm{O}-\mathrm{CH}_{2}-\mathrm{CH}=\mathrm{CH}_{2}$ side chains have very different conformations (Fig. 1), which may be attributable, at least in part, to an intramolecular $\mathrm{C} 12-\mathrm{H} 12 A \cdots \mathrm{O} 3$ interaction (Table 1 ). The molecules form $\pi-\pi$ stacks in the crystal structure (Fig. 2), with alternating centroid-to-centroid

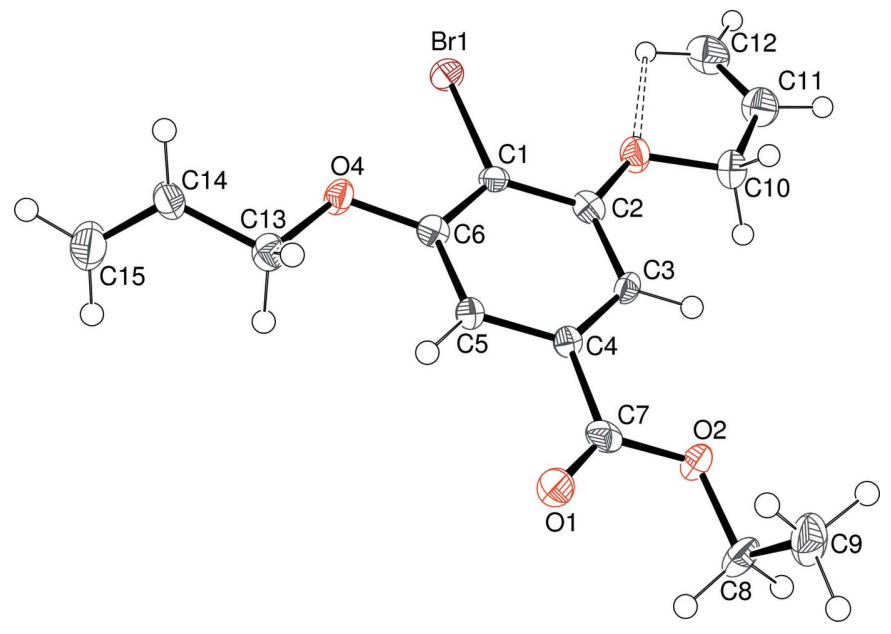

Figure 1

The molecular structure of (I), showing 50\% displacement ellipsoids for non-H atoms. The intramolecular $\mathrm{C}-\mathrm{H} \cdots \mathrm{O}$ interaction referred to in the Comment is indicated by a dashed line.
Received 16 January 2007 Accepted 16 January 2007
(C) 2007 International Union of Crystallography All rights reserved 


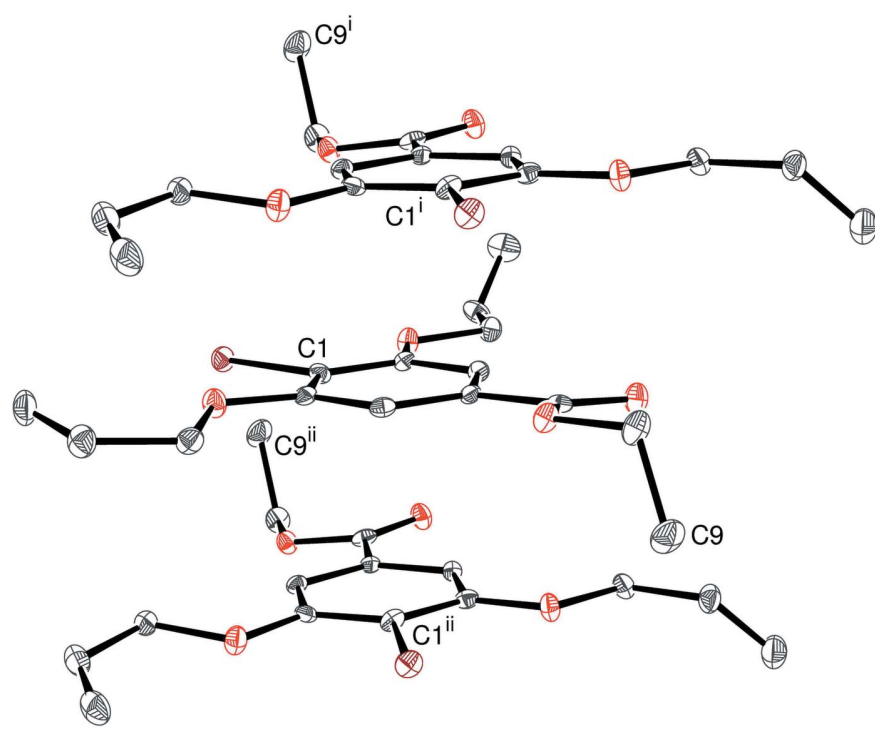

Figure 2

Part of a $\pi-\pi$ stacked column of molecules ( $30 \%$ displacement ellipsoids and $\mathrm{H}$ atoms omitted). [Symmetry codes: (i) $x,-y, 1-z$; (ii) $x, 1-y$, $1-z$.]

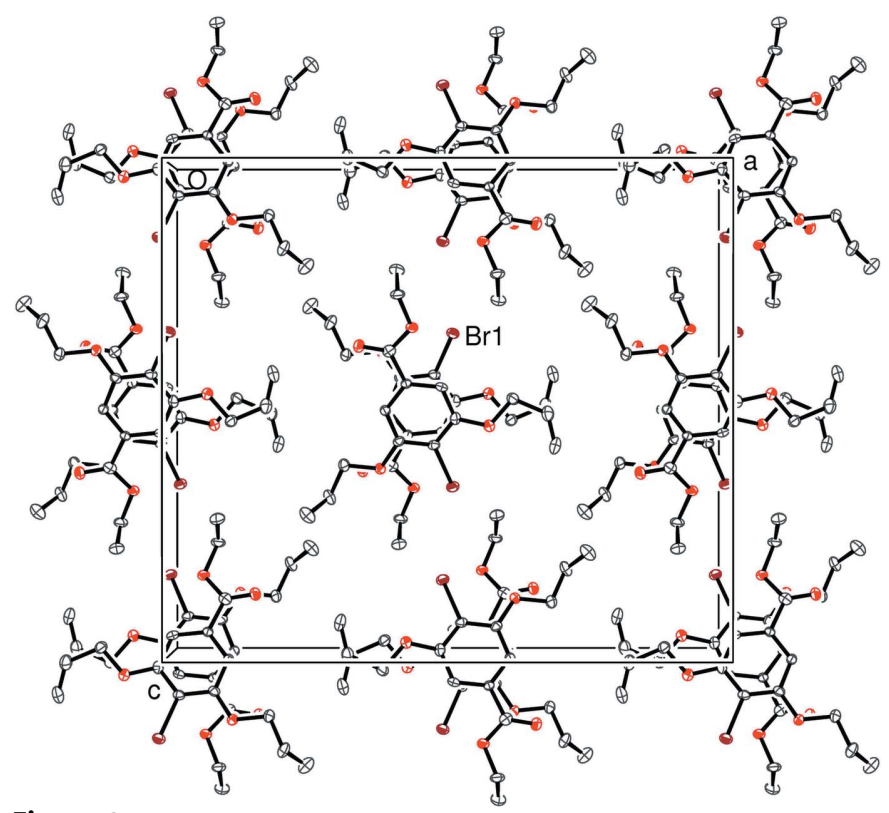

Figure 3

Unit-cell contents of (I), viewed down [010] (50\% displacement ellipsoids and $\mathrm{H}$ atoms omitted).

separations between benzene rings $\left[C g \cdots C g^{\mathrm{i}}=3.626\right.$ (2), $C g \cdots C g^{\mathrm{ii}}=3.466$ (2) $\AA$; symmetry codes: (i) $x,-y, 1-z$; (ii) $x$, $1-y, 1-z]$. The stacking interactions give rise to columns of molecules along [010] (Fig. 3).

\section{Experimental}

4-Bromo-3,5-dihydroxybenzoic acid $(6.8 \mathrm{~g}, 0.03 \mathrm{~mol})$ was added to $100 \mathrm{ml}$ of ethanol. Concentrated $\mathrm{H}_{2} \mathrm{SO}_{4}(1 \mathrm{ml})$ was added and the mixture was refluxed for $14 \mathrm{~h}$. After cooling, the solvent was removed at reduced pressure to give a pale yellow oil. Diethyl ether $(100 \mathrm{ml})$ was added and the mixture was neutralized by careful addition of a saturated $\mathrm{NaHCO}_{3}$ solution $(100 \mathrm{ml})$. The mixture was transferred to a separating funnel and the product extracted with diethyl ether $(4 \times$ $100 \mathrm{ml}$ ). The combined extracts were dried over anhydrous $\mathrm{MgSO}_{4}$ and evaporated under reduced pressure to give 4-bromo-3,5-dihydroxybenzoic acid ethyl ester as a white powder $(7.5 \mathrm{~g}, 96 \%)$. Ethyl 4-bromo-3,5-dihydroxybenzoate $(3.00 \mathrm{~g}, 0.011 \mathrm{~mol})$, allyl bromide $(1.30 \mathrm{~g}, 0.011 \mathrm{~mol})$ and $\mathrm{K}_{2} \mathrm{CO}_{3}(8.00 \mathrm{~g}, 0.0579 \mathrm{~mol})$ were added to $100 \mathrm{ml}$ of dry acetone. The mixture was stirred at room temperature under a nitrogen atmosphere for $14 \mathrm{~h}$, then filtered and the solvent removed at reduced pressure to give a dark brown oil. Thin layer chromatography (4:1 hexane-ethyl acetate eluent) showed the title compound as a sharp spot at $R_{\mathrm{F}}=0.52$. The crude product was purified by flash column chromatography to yield a white powder $(1.42 \mathrm{~g}, 38 \%)$. A sample of this powder was recrystallized from hot hexane to give translucent needles of (I) (m.p. 315-317 K).

Crystal data

$\mathrm{C}_{15} \mathrm{H}_{17} \mathrm{BrO}_{4}$

$M_{r}=341.20$

Orthorhombic, $C 222_{1}$

$a=22.1421$ (2) А

$b=7.0559$ (13) $\AA$

$c=19.5604(11) \AA$

$V=3056.0(6) \AA^{3}$

$Z=8$

$D_{x}=1.483 \mathrm{Mg} \mathrm{m}^{-3}$

Mo $K \alpha$ radiation

$\mu=2.70 \mathrm{~mm}^{-1}$

$T=120(2) \mathrm{K}$

Needle, colourless

$0.22 \times 0.04 \times 0.02 \mathrm{~mm}$

\section{Data collection}

Nonius KappaCCD diffractometer

$\omega$ and $\varphi$ scans

Absorption correction: multi-scan

(SORTAV; Blessing, 1995)

$T_{\min }=0.588, T_{\max }=0.948$

10933 measured reflections 3495 independent reflections 2604 reflections with $I>2 \sigma(I)$ $R_{\text {int }}=0.084$

$\theta_{\max }=27.5^{\circ}$

Refinement

Refinement on $F^{2}$

$R\left[F^{2}>2 \sigma\left(F^{2}\right)\right]=0.052$

$w R\left(F^{2}\right)=0.084$

$S=1.01$

3495 reflections

183 parameters

$\mathrm{H}$-atom parameters constrained

$$
\begin{aligned}
& w=1 /\left[\sigma^{2}\left(F_{\mathrm{o}}^{2}\right)+(0.0143 P)^{2}\right] \\
& \text { where } P=\left(F_{\mathrm{o}}^{2}+2 F_{\mathrm{c}}^{2}\right) / 3 \\
& (\Delta / \sigma)_{\max }<0.001 \\
& \Delta \rho_{\max }=0.47 \mathrm{e} \AA^{-3} \\
& \Delta \rho_{\min }=-0.53 \mathrm{e}^{-3} \\
& \text { Absolute structure: Flack (1983), } \\
& \text { 1500 Friedel pairs } \\
& \text { Flack parameter: } 0.106(13)
\end{aligned}
$$

Table 1

Hydrogen-bond geometry $\left(\AA,{ }^{\circ}\right)$.

\begin{tabular}{lllll}
\hline$D-\mathrm{H} \cdots A$ & $D-\mathrm{H}$ & $\mathrm{H} \cdots A$ & $D \cdots A$ & $D-\mathrm{H} \cdots A$ \\
\hline $\mathrm{C} 12-\mathrm{H} 12 A \cdots \mathrm{O} 3$ & 0.95 & 2.39 & $2.715(6)$ & 100 \\
\hline
\end{tabular}

$\mathrm{H}$ atoms were placed in idealized locations $(\mathrm{C}-\mathrm{H}=0.95-0.99 \AA)$ and refined as riding atoms, with $U_{\text {iso }}(\mathrm{H})=1.2 U_{\text {eq }}(\mathrm{C})$ or $1.5 U_{\text {eq }}$ (methyl C).

Data collection: COLLECT (Nonius, 1998); cell refinement: SCALEPACK (Otwinowski \& Minor, 1997); data reduction: SCALEPACK and DENZO (Otwinowski \& Minor, 1997); program(s) used to solve structure: SHELXS97 (Sheldrick, 1997); program(s) used to refine structure: SHELXL97 (Sheldrick, 1997); molecular graphics: ORTEP-3 (Farrugia, 1997); software used to prepare material for publication: SHELXL97.

We thank the EPSRC UK National Crystallography Service (University of Southampton) for the data collection. 


\section{organic papers}

\section{References}

Blessing, R. H. (1995). Acta Cryst. A51, 33-38.

Farrugia, L. J. (1997). J. Appl. Cryst. 30, 565.

Flack, H. D. (1983). Acta Cryst. A39, 876-881.

Kirsop, P., Storey, J. M. D. \& Harrison, W. T. A. (2004). Acta Cryst. E60, o1147o1148.
Nonius (1998). COLLECT. Nonius BV, Delft, The Netherlands.

Otwinowski, Z. \& Minor, W. (1997). Methods in Enzymology, Vol. 276,

Macromolecular Crystallography, Part A, edited by C. W. Carter Jr \& R. M.

Sweet, pp. 307-326. New York: Academic Press.

Sheldrick, G. M. (1997). SHELXS97 and SHELXL97. University of Göttingen, Germany. 


\section{supporting information}

Acta Cryst. (2007). E63, o833-o835 [https://doi.org/10.1107/S1600536807002383]

Ethyl 3,5-bis(allyloxy)-4-bromobenzoate

Peter Kirsop, John M. D. Storey and William T. A. Harrison

Ethyl 3,5-bis(allyloxy)-4-bromobenzoate

Crystal data

$\mathrm{C}_{15} \mathrm{H}_{17} \mathrm{BrO}_{4}$

$M_{r}=341.20$

Orthorhombic, $C 222_{1}$

Hall symbol: C 2c 2

$a=22.1421(2) \AA$

$b=7.0559(13) \AA$

$c=19.5604(11) \AA$

$V=3056.0(6) \AA^{3}$

$Z=8$

$F(000)=1392$

$D_{\mathrm{x}}=1.483 \mathrm{Mg} \mathrm{m}^{-3}$

Mo $K \alpha$ radiation, $\lambda=0.71073 \AA$

Cell parameters from 1957 reflections

$\theta=2.9-27.5^{\circ}$

$\mu=2.70 \mathrm{~mm}^{-1}$

$T=120 \mathrm{~K}$

Needle, colourless

$0.22 \times 0.04 \times 0.02 \mathrm{~mm}$

\section{Data collection}

Nonius KappaCCD

diffractometer

Radiation source: fine-focus sealed tube

Graphite monochromator

$\omega$ and $\varphi$ scans

Absorption correction: multi-scan

(SORTAV; Blessing, 1995)

$T_{\text {min }}=0.588, T_{\text {max }}=0.948$

10933 measured reflections

3495 independent reflections

2604 reflections with $I>2 \sigma(I)$

$R_{\text {int }}=0.084$

$\theta_{\max }=27.5^{\circ}, \theta_{\min }=3.0^{\circ}$

$h=-20 \rightarrow 28$

$k=-9 \rightarrow 9$

$l=-24 \rightarrow 25$

Hydrogen site location: inferred from

neighbouring sites

$\mathrm{H}$-atom parameters constrained

$w=1 /\left[\sigma^{2}\left(F_{0}^{2}\right)+(0.0143 P)^{2}\right]$

where $P=\left(F_{\mathrm{o}}^{2}+2 F_{\mathrm{c}}{ }^{2}\right) / 3$

$(\Delta / \sigma)_{\max }<0.001$

$\Delta \rho_{\max }=0.47 \mathrm{e} \AA^{-3}$

$\Delta \rho_{\min }=-0.53$ e $\AA^{-3}$

Absolute structure: Flack (1983), 1500 Friedel pairs

Absolute structure parameter: 0.106 (13)

Secondary atom site location: difference Fourier

map

Special details

Geometry. All e.s.d.'s (except the e.s.d. in the dihedral angle between two 1.s. planes) are estimated using the full covariance matrix. The cell e.s.d.'s are taken into account individually in the estimation of e.s.d.'s in distances, angles and torsion angles; correlations between e.s.d.'s in cell parameters are only used when they are defined by crystal symmetry. An approximate (isotropic) treatment of cell e.s.d.'s is used for estimating e.s.d.'s involving l.s. planes. 
Refinement. Refinement of $F^{2}$ against ALL reflections. The weighted $R$-factor $w R$ and goodness of fit $S$ are based on $F^{2}$, conventional $R$-factors $R$ are based on $F$, with $F$ set to zero for negative $F^{2}$. The threshold expression of $F^{2}>\sigma\left(F^{2}\right)$ is used only for calculating $R$-factors(gt) etc. and is not relevant to the choice of reflections for refinement. $R$-factors based on $F^{2}$ are statistically about twice as large as those based on $F$, and $R$ - factors based on ALL data will be even larger.

Fractional atomic coordinates and isotropic or equivalent isotropic displacement parameters $\left(\hat{A}^{2}\right)$

\begin{tabular}{|c|c|c|c|c|}
\hline & $x$ & $y$ & $z$ & $U_{\text {iso }} * / U_{\text {eq }}$ \\
\hline $\mathrm{C} 1$ & $0.4754(2)$ & $0.3097(5)$ & $0.4289(2)$ & $0.0184(11)$ \\
\hline $\mathrm{C} 2$ & $0.5137(2)$ & $0.2603(5)$ & $0.4827(2)$ & $0.0185(10)$ \\
\hline $\mathrm{C} 3$ & $0.4891(2)$ & $0.2071(5)$ & $0.5457(2)$ & $0.0173(10)$ \\
\hline $\mathrm{H} 3$ & 0.5145 & 0.1745 & 0.5829 & $0.021 *$ \\
\hline $\mathrm{C} 4$ & $0.4267(2)$ & $0.2030(6)$ & $0.5526(2)$ & $0.0159(10)$ \\
\hline $\mathrm{C} 5$ & 0.3893 (2) & $0.2505(6)$ & $0.4987(2)$ & $0.0171(10)$ \\
\hline H5 & 0.3467 & 0.2467 & 0.5046 & $0.021 *$ \\
\hline C6 & $0.4132(2)$ & $0.3035(6)$ & $0.4364(2)$ & $0.0184(11)$ \\
\hline $\mathrm{C} 7$ & $0.3970(2)$ & $0.1440(6)$ & $0.6178(2)$ & $0.0215(11)$ \\
\hline $\mathrm{C} 8$ & $0.4125(2)$ & $0.0183(7)$ & $0.7297(2)$ & $0.0278(12)$ \\
\hline H8A & 0.4379 & -0.0848 & 0.7484 & $0.033 *$ \\
\hline H8B & 0.3713 & -0.0323 & 0.7227 & $0.033 *$ \\
\hline $\mathrm{C} 9$ & $0.4103(2)$ & $0.1796(7)$ & $0.7799(2)$ & $0.0360(14)$ \\
\hline H9A & 0.3918 & 0.1362 & 0.8226 & $0.054^{*}$ \\
\hline H9B & 0.3863 & 0.2835 & 0.7607 & $0.054 *$ \\
\hline $\mathrm{H} 9 \mathrm{C}$ & 0.4514 & 0.2244 & 0.7891 & $0.054 *$ \\
\hline $\mathrm{C} 10$ & $0.6157(2)$ & $0.2438(7)$ & $0.5223(2)$ & $0.0246(12)$ \\
\hline H10A & 0.6095 & 0.1206 & 0.5454 & $0.030 *$ \\
\hline H10B & 0.6102 & 0.3462 & 0.5563 & $0.030 *$ \\
\hline C11 & $0.6776(2)$ & $0.2528(7)$ & $0.4924(3)$ & $0.0313(12)$ \\
\hline H11 & 0.7105 & 0.2476 & 0.5236 & $0.038 *$ \\
\hline $\mathrm{C} 12$ & $0.6911(2)$ & $0.2671(7)$ & 0.4278 & $0.0360(13)$ \\
\hline $\mathrm{H} 12 \mathrm{~A}$ & 0.6598 & 0.2728 & 0.3946 & $0.043 *$ \\
\hline H12B & 0.7321 & 0.2719 & 0.4138 & $0.043 *$ \\
\hline C13 & $0.3152(2)$ & $0.3480(7)$ & $0.3880(2)$ & $0.0235(11)$ \\
\hline H13A & 0.3021 & 0.4327 & 0.4255 & $0.028 *$ \\
\hline H13B & 0.3020 & 0.2174 & 0.3988 & $0.028 *$ \\
\hline $\mathrm{C} 14$ & $0.2884(2)$ & $0.4107(6)$ & $0.3224(2)$ & $0.0274(12)$ \\
\hline H14 & 0.2995 & 0.5312 & 0.3048 & $0.033 *$ \\
\hline $\mathrm{C} 15$ & $0.2503(2)$ & $0.3082(7)$ & $0.2876(3)$ & $0.0383(15)$ \\
\hline $\mathrm{H} 15 \mathrm{~A}$ & 0.2385 & 0.1871 & 0.3042 & $0.046^{*}$ \\
\hline H15B & 0.2343 & 0.3545 & 0.2458 & $0.046^{*}$ \\
\hline $\mathrm{O} 1$ & $0.34357(14)$ & $0.1487(5)$ & $0.62781(15)$ & $0.0253(8)$ \\
\hline $\mathrm{O} 2$ & $0.43720(12)$ & $0.0814(4)$ & $0.66439(15)$ & $0.0216(7)$ \\
\hline $\mathrm{O} 3$ & $0.57368(14)$ & $0.2653(4)$ & $0.46792(16)$ & $0.0251(8)$ \\
\hline $\mathrm{O} 4$ & $0.37974(13)$ & $0.3545(4)$ & $0.38091(14)$ & $0.0220(7)$ \\
\hline $\mathrm{Br} 1$ & $0.509124(19)$ & $0.38018(6)$ & $0.34421(2)$ & $0.02348(13)$ \\
\hline
\end{tabular}


Atomic displacement parameters $\left(\AA^{2}\right)$

\begin{tabular}{lllllll}
\hline & $U^{11}$ & $U^{22}$ & $U^{33}$ & $U^{12}$ & $U^{13}$ & $U^{23}$ \\
\hline C1 & $0.023(3)$ & $0.017(2)$ & $0.015(2)$ & $-0.0058(18)$ & $0.001(2)$ & $0.0005(18)$ \\
C2 & $0.017(3)$ & $0.014(2)$ & $0.025(3)$ & $0.003(2)$ & $0.001(2)$ & $-0.0052(17)$ \\
C3 & $0.024(3)$ & $0.0149(19)$ & $0.013(2)$ & $0.0015(19)$ & $-0.004(2)$ & $-0.0008(16)$ \\
C4 & $0.017(3)$ & $0.016(2)$ & $0.014(3)$ & $0.0011(18)$ & $0.001(2)$ & $-0.0034(18)$ \\
C5 & $0.016(3)$ & $0.018(2)$ & $0.017(3)$ & $0.0009(19)$ & $-0.001(2)$ & $-0.001(2)$ \\
C6 & $0.021(3)$ & $0.019(3)$ & $0.016(3)$ & $-0.0008(18)$ & $0.003(2)$ & $-0.0041(19)$ \\
C7 & $0.027(3)$ & $0.015(2)$ & $0.023(3)$ & $-0.006(2)$ & $0.002(2)$ & $-0.006(2)$ \\
C8 & $0.032(3)$ & $0.037(3)$ & $0.014(3)$ & $0.005(2)$ & $0.002(2)$ & $0.005(2)$ \\
C9 & $0.036(3)$ & $0.052(4)$ & $0.021(3)$ & $0.000(2)$ & $-0.003(3)$ & $-0.009(2)$ \\
C10 & $0.023(3)$ & $0.029(3)$ & $0.022(3)$ & $0.003(2)$ & $-0.003(2)$ & $-0.004(2)$ \\
C11 & $0.023(3)$ & $0.033(3)$ & $0.038(3)$ & $0.005(2)$ & $-0.002(3)$ & $0.002(3)$ \\
C12 & $0.020(3)$ & $0.041(3)$ & $0.046(4)$ & $0.001(2)$ & $0.008(3)$ & $0.001(3)$ \\
C13 & $0.019(3)$ & $0.025(3)$ & $0.026(3)$ & $0.004(2)$ & $-0.001(2)$ & $0.001(2)$ \\
C14 & $0.023(3)$ & $0.024(3)$ & $0.035(3)$ & $-0.004(2)$ & $-0.010(2)$ & $0.002(2)$ \\
C15 & $0.038(4)$ & $0.038(3)$ & $0.040(4)$ & $0.002(2)$ & $-0.015(3)$ & $0.002(3)$ \\
O1 & $0.0175(19)$ & $0.033(2)$ & $0.0253(18)$ & $0.0034(16)$ & $0.0019(14)$ & $0.0034(16)$ \\
O2 & $0.0218(17)$ & $0.0273(17)$ & $0.0157(17)$ & $0.0050(12)$ & $-0.0004(14)$ & $0.0030(15)$ \\
O3 & $0.019(2)$ & $0.0346(19)$ & $0.0217(19)$ & $-0.0019(14)$ & $-0.0013(17)$ & $-0.0002(16)$ \\
O4 & $0.0168(18)$ & $0.0308(18)$ & $0.0184(17)$ & $0.0035(16)$ & $-0.0010(13)$ & $0.0049(15)$ \\
Br1 & $0.0254(2)$ & $0.0274(2)$ & $0.0176(2)$ & $-0.0024(2)$ & $0.0027(2)$ & $0.0011(2)$ \\
& & & & & & \\
\hline
\end{tabular}

Geometric parameters $\left(A,{ }^{\circ}\right)$

\begin{tabular}{llll}
\hline $\mathrm{C} 1-\mathrm{C} 6$ & $1.386(6)$ & $\mathrm{C} 9-\mathrm{H} 9 \mathrm{~B}$ & 0.980 \\
$\mathrm{C} 1-\mathrm{C} 2$ & $1.395(6)$ & $\mathrm{C} 9-\mathrm{H} 9 \mathrm{C}$ & 0.980 \\
$\mathrm{C} 1-\mathrm{Br} 1$ & $1.884(4)$ & $\mathrm{C} 10-\mathrm{O} 3$ & $1.421(5)$ \\
$\mathrm{C} 2-\mathrm{O} 3$ & $1.361(5)$ & $\mathrm{C} 10-\mathrm{C} 11$ & $1.492(6)$ \\
$\mathrm{C} 2-\mathrm{C} 3$ & $1.397(6)$ & $\mathrm{C} 10-\mathrm{H} 10 \mathrm{~A}$ & 0.990 \\
$\mathrm{C} 3-\mathrm{C} 4$ & $1.389(6)$ & $\mathrm{C} 10-\mathrm{H} 10 \mathrm{~B}$ & 0.990 \\
$\mathrm{C} 3-\mathrm{H} 3$ & 0.950 & $\mathrm{C} 11-\mathrm{C} 12$ & $1.303(6)$ \\
$\mathrm{C} 4-\mathrm{C} 5$ & $1.381(6)$ & $\mathrm{C} 11-\mathrm{H} 11$ & 0.950 \\
$\mathrm{C} 4-\mathrm{C} 7$ & $1.494(6)$ & $\mathrm{C} 12-\mathrm{H} 12 \mathrm{~A}$ & 0.950 \\
$\mathrm{C} 5-\mathrm{C} 6$ & $1.381(6)$ & $\mathrm{C} 12-\mathrm{H} 12 \mathrm{~B}$ & 0.950 \\
$\mathrm{C} 5-\mathrm{H} 5$ & 0.950 & $\mathrm{C} 13-\mathrm{O} 4$ & $1.436(5)$ \\
$\mathrm{C} 6-\mathrm{O} 4$ & $1.363(5)$ & $\mathrm{C} 13-\mathrm{C} 14$ & $1.481(6)$ \\
$\mathrm{C} 7-\mathrm{O} 1$ & $1.200(5)$ & $\mathrm{C} 13-\mathrm{H} 13 \mathrm{~A}$ & 0.990 \\
$\mathrm{C} 7-\mathrm{O} 2$ & $1.348(5)$ & $\mathrm{C} 13-\mathrm{H} 13 \mathrm{~B}$ & 0.990 \\
$\mathrm{C} 8-\mathrm{O} 2$ & $1.459(5)$ & $\mathrm{C} 14-\mathrm{C} 15$ & $1.303(6)$ \\
$\mathrm{C} 8-\mathrm{C} 9$ & $1.504(6)$ & $\mathrm{C} 14-\mathrm{H} 14$ & 0.950 \\
$\mathrm{C} 8-\mathrm{H} 8 \mathrm{~A}$ & 0.990 & $\mathrm{C} 15-\mathrm{H} 15 \mathrm{~A}$ & 0.950 \\
$\mathrm{C} 8-\mathrm{H} 8 \mathrm{~B}$ & 0.990 & $\mathrm{C} 15-\mathrm{H} 15 \mathrm{~B}$ & 0.950 \\
$\mathrm{C} 9-\mathrm{H} 9 \mathrm{~A}$ & 0.980 & & 109.5 \\
$\mathrm{C} 6-\mathrm{C} 1-\mathrm{C} 2$ & & $\mathrm{C} 8-\mathrm{C} 9-\mathrm{H} 9 \mathrm{C}$ & 109.5 \\
$\mathrm{C} 6-\mathrm{C} 1-\mathrm{Br} 1$ & $121.0(4)$ & $\mathrm{H} 9 \mathrm{~A}-\mathrm{C} 9-\mathrm{H} 9 \mathrm{C}$ &
\end{tabular}




\begin{tabular}{|c|c|c|c|}
\hline $\mathrm{C} 2-\mathrm{C} 1-\mathrm{Br} 1$ & $119.2(3)$ & $\mathrm{H} 9 \mathrm{~B}-\mathrm{C} 9-\mathrm{H} 9 \mathrm{C}$ & 109.5 \\
\hline $\mathrm{O} 3-\mathrm{C} 2-\mathrm{C} 1$ & $115.2(4)$ & $\mathrm{O} 3-\mathrm{C} 10-\mathrm{C} 11$ & $107.7(4)$ \\
\hline $\mathrm{O} 3-\mathrm{C} 2-\mathrm{C} 3$ & $125.1(4)$ & $\mathrm{O} 3-\mathrm{C} 10-\mathrm{H} 10 \mathrm{~A}$ & 110.2 \\
\hline $\mathrm{C} 1-\mathrm{C} 2-\mathrm{C} 3$ & $119.7(4)$ & $\mathrm{C} 11-\mathrm{C} 10-\mathrm{H} 10 \mathrm{~A}$ & 110.2 \\
\hline $\mathrm{C} 4-\mathrm{C} 3-\mathrm{C} 2$ & $118.6(4)$ & $\mathrm{O} 3-\mathrm{C} 10-\mathrm{H} 10 \mathrm{~B}$ & 110.2 \\
\hline $\mathrm{C} 4-\mathrm{C} 3-\mathrm{H} 3$ & 120.7 & $\mathrm{C} 11-\mathrm{C} 10-\mathrm{H} 10 \mathrm{~B}$ & 110.2 \\
\hline $\mathrm{C} 2-\mathrm{C} 3-\mathrm{H} 3$ & 120.7 & $\mathrm{H} 10 \mathrm{~A}-\mathrm{C} 10-\mathrm{H} 10 \mathrm{~B}$ & 108.5 \\
\hline $\mathrm{C} 5-\mathrm{C} 4-\mathrm{C} 3$ & $121.1(4)$ & $\mathrm{C} 12-\mathrm{C} 11-\mathrm{C} 10$ & $126.3(5)$ \\
\hline $\mathrm{C} 5-\mathrm{C} 4-\mathrm{C} 7$ & $117.1(4)$ & $\mathrm{C} 12-\mathrm{C} 11-\mathrm{H} 11$ & 116.8 \\
\hline $\mathrm{C} 3-\mathrm{C} 4-\mathrm{C} 7$ & $121.7(4)$ & $\mathrm{C} 10-\mathrm{C} 11-\mathrm{H} 11$ & 116.8 \\
\hline $\mathrm{C} 4-\mathrm{C} 5-\mathrm{C} 6$ & $120.6(4)$ & $\mathrm{C} 11-\mathrm{C} 12-\mathrm{H} 12 \mathrm{~A}$ & 120.0 \\
\hline $\mathrm{C} 4-\mathrm{C} 5-\mathrm{H} 5$ & 119.7 & $\mathrm{C} 11-\mathrm{C} 12-\mathrm{H} 12 \mathrm{~B}$ & 120.0 \\
\hline $\mathrm{C} 6-\mathrm{C} 5-\mathrm{H} 5$ & 119.7 & $\mathrm{H} 12 \mathrm{~A}-\mathrm{C} 12-\mathrm{H} 12 \mathrm{~B}$ & 120.0 \\
\hline $\mathrm{O} 4-\mathrm{C} 6-\mathrm{C} 5$ & $124.5(4)$ & $\mathrm{O} 4-\mathrm{C} 13-\mathrm{C} 14$ & $107.8(4)$ \\
\hline $\mathrm{O} 4-\mathrm{C} 6-\mathrm{C} 1$ & $116.6(4)$ & $\mathrm{O} 4-\mathrm{C} 13-\mathrm{H} 13 \mathrm{~A}$ & 110.1 \\
\hline $\mathrm{C} 5-\mathrm{C} 6-\mathrm{C} 1$ & $118.9(4)$ & $\mathrm{C} 14-\mathrm{C} 13-\mathrm{H} 13 \mathrm{~A}$ & 110.1 \\
\hline $\mathrm{O} 1-\mathrm{C} 7-\mathrm{O} 2$ & $123.4(4)$ & $\mathrm{O} 4-\mathrm{C} 13-\mathrm{H} 13 \mathrm{~B}$ & 110.1 \\
\hline $\mathrm{O} 1-\mathrm{C} 7-\mathrm{C} 4$ & $124.4(4)$ & $\mathrm{C} 14-\mathrm{C} 13-\mathrm{H} 13 \mathrm{~B}$ & 110.1 \\
\hline $\mathrm{O} 2-\mathrm{C} 7-\mathrm{C} 4$ & $112.3(4)$ & $\mathrm{H} 13 \mathrm{~A}-\mathrm{C} 13-\mathrm{H} 13 \mathrm{~B}$ & 108.5 \\
\hline $\mathrm{O} 2-\mathrm{C} 8-\mathrm{C} 9$ & $110.6(4)$ & $\mathrm{C} 15-\mathrm{C} 14-\mathrm{C} 13$ & $123.1(5)$ \\
\hline $\mathrm{O} 2-\mathrm{C} 8-\mathrm{H} 8 \mathrm{~A}$ & 109.5 & $\mathrm{C} 15-\mathrm{C} 14-\mathrm{H} 14$ & 118.5 \\
\hline $\mathrm{C} 9-\mathrm{C} 8-\mathrm{H} 8 \mathrm{~A}$ & 109.5 & $\mathrm{C} 13-\mathrm{C} 14-\mathrm{H} 14$ & 118.5 \\
\hline $\mathrm{O} 2-\mathrm{C} 8-\mathrm{H} 8 \mathrm{~B}$ & 109.5 & $\mathrm{C} 14-\mathrm{C} 15-\mathrm{H} 15 \mathrm{~A}$ & 120.0 \\
\hline $\mathrm{C} 9-\mathrm{C} 8-\mathrm{H} 8 \mathrm{~B}$ & 109.5 & $\mathrm{C} 14-\mathrm{C} 15-\mathrm{H} 15 \mathrm{~B}$ & 120.0 \\
\hline $\mathrm{H} 8 \mathrm{~A}-\mathrm{C} 8-\mathrm{H} 8 \mathrm{~B}$ & 108.1 & $\mathrm{H} 15 \mathrm{~A}-\mathrm{C} 15-\mathrm{H} 15 \mathrm{~B}$ & 120.0 \\
\hline $\mathrm{C} 8-\mathrm{C} 9-\mathrm{H} 9 \mathrm{~A}$ & 109.5 & $\mathrm{C} 7-\mathrm{O} 2-\mathrm{C} 8$ & $116.4(3)$ \\
\hline $\mathrm{C} 8-\mathrm{C} 9-\mathrm{H} 9 \mathrm{~B}$ & 109.5 & $\mathrm{C} 2-\mathrm{O} 3-\mathrm{C} 10$ & $118.5(3)$ \\
\hline $\mathrm{H} 9 \mathrm{~A}-\mathrm{C} 9-\mathrm{H} 9 \mathrm{~B}$ & 109.5 & $\mathrm{C} 6-\mathrm{O} 4-\mathrm{C} 13$ & $117.1(3)$ \\
\hline $\mathrm{C} 6-\mathrm{C} 1-\mathrm{C} 2-\mathrm{O} 3$ & $177.6(3)$ & $\mathrm{C} 5-\mathrm{C} 4-\mathrm{C} 7-\mathrm{O} 1$ & $-5.7(6)$ \\
\hline $\mathrm{Br} 1-\mathrm{C} 1-\mathrm{C} 2-\mathrm{O} 3$ & $-1.0(5)$ & $\mathrm{C} 3-\mathrm{C} 4-\mathrm{C} 7-\mathrm{O} 1$ & $175.3(4)$ \\
\hline $\mathrm{C} 6-\mathrm{C} 1-\mathrm{C} 2-\mathrm{C} 3$ & $-1.2(6)$ & $\mathrm{C} 5-\mathrm{C} 4-\mathrm{C} 7-\mathrm{O} 2$ & $173.3(4)$ \\
\hline $\mathrm{Br} 1-\mathrm{C} 1-\mathrm{C} 2-\mathrm{C} 3$ & $-179.7(3)$ & $\mathrm{C} 3-\mathrm{C} 4-\mathrm{C} 7-\mathrm{O} 2$ & $-5.6(6)$ \\
\hline $\mathrm{O} 3-\mathrm{C} 2-\mathrm{C} 3-\mathrm{C} 4$ & $-177.9(3)$ & $\mathrm{O} 3-\mathrm{C} 10-\mathrm{C} 11-\mathrm{C} 12$ & $-3.5(7)$ \\
\hline $\mathrm{C} 1-\mathrm{C} 2-\mathrm{C} 3-\mathrm{C} 4$ & $0.7(5)$ & $\mathrm{O} 4-\mathrm{C} 13-\mathrm{C} 14-\mathrm{C} 15$ & $123.9(5)$ \\
\hline $\mathrm{C} 2-\mathrm{C} 3-\mathrm{C} 4-\mathrm{C} 5$ & $-0.1(6)$ & $\mathrm{O} 1-\mathrm{C} 7-\mathrm{O} 2-\mathrm{C} 8$ & $-0.3(6)$ \\
\hline $\mathrm{C} 2-\mathrm{C} 3-\mathrm{C} 4-\mathrm{C} 7$ & $178.8(4)$ & $\mathrm{C} 4-\mathrm{C} 7-\mathrm{O} 2-\mathrm{C} 8$ & $-179.3(3)$ \\
\hline $\mathrm{C} 3-\mathrm{C} 4-\mathrm{C} 5-\mathrm{C} 6$ & $0.0(7)$ & $\mathrm{C} 9-\mathrm{C} 8-\mathrm{O} 2-\mathrm{C} 7$ & $-91.1(5)$ \\
\hline $\mathrm{C} 7-\mathrm{C} 4-\mathrm{C} 5-\mathrm{C} 6$ & $-179.0(4)$ & $\mathrm{C} 1-\mathrm{C} 2-\mathrm{O} 3-\mathrm{C} 10$ & $171.0(4)$ \\
\hline $\mathrm{C} 4-\mathrm{C} 5-\mathrm{C} 6-\mathrm{O} 4$ & $-179.3(4)$ & $\mathrm{C} 3-\mathrm{C} 2-\mathrm{O} 3-\mathrm{C} 10$ & $-10.3(6)$ \\
\hline $\mathrm{C} 4-\mathrm{C} 5-\mathrm{C} 6-\mathrm{C} 1$ & $-0.5(6)$ & $\mathrm{C} 11-\mathrm{C} 10-\mathrm{O} 3-\mathrm{C} 2$ & $179.5(3)$ \\
\hline $\mathrm{C} 2-\mathrm{C} 1-\mathrm{C} 6-\mathrm{O} 4$ & $180.0(4)$ & $\mathrm{C} 5-\mathrm{C} 6-\mathrm{O} 4-\mathrm{C} 13$ & $-1.4(6)$ \\
\hline $\mathrm{Br} 1-\mathrm{C} 1-\mathrm{C} 6-\mathrm{O} 4$ & $-1.5(5)$ & $\mathrm{C} 1-\mathrm{C} 6-\mathrm{O} 4-\mathrm{C} 13$ & $179.8(4)$ \\
\hline $\mathrm{C} 2-\mathrm{C} 1-\mathrm{C} 6-\mathrm{C} 5$ & $1.1(6)$ & $\mathrm{C} 14-\mathrm{C} 13-\mathrm{O} 4-\mathrm{C} 6$ & $179.3(3)$ \\
\hline $\mathrm{Br} 1-\mathrm{C} 1-\mathrm{C} 6-\mathrm{C} 5$ & $179.6(3)$ & & \\
\hline
\end{tabular}


Hydrogen-bond geometry $\left(A,{ }^{\circ}\right)$

\begin{tabular}{lllll}
\hline$D-\mathrm{H} \cdots A$ & $D-\mathrm{H}$ & $\mathrm{H} \cdots A$ & $D \cdots A$ & $D-\mathrm{H} \cdots A$ \\
\hline $\mathrm{C} 12-\mathrm{H} 12 A \cdots \mathrm{O} 3$ & 0.95 & 2.39 & $2.715(6)$ & 100 \\
\hline
\end{tabular}

